\title{
Avaliação dos Fatores Ambientais no Desenvolvimento Corporal de Cordeiros Deslanados da Raça Morada Nova
}

\author{
Antônio Amaury Oriá Fernandes ${ }^{1}$, David Buchanan², Arturo Bernardo Selaive-Villarroel ${ }^{3}$
}

RESUMO -Foram analisados registros de um rebanho de ovinos da raça Morada Nova, coletados num período de 11 anos (1981 a 1991), com o objetivo de avaliar os efeitos de ambiente sobre as características de crescimento dos cordeiros. Os efeitos de ano de nascimento (A), sexo do cordeiro (S), tipo de nascimento $(\mathrm{T})$ e peso da ovelha ao parto (P) foram importantes fontes de variação para explicar as diferenças nos pesos corporais ao nascimento (PN), ao desmame (PD), aos seis meses (PM6) e aos 12 meses de idade (PM12). Os cordeiros machos foram mais pesados que as fêmeas em todas as idades estudadas. Cordeiros nascidos e criados como simples (SS) pesaram mais que os nascidos duplos e criados como simples (GS) e os nascidos e criados como duplos (GG). A idade da ovelha (I) teve efeito significativo sobre PN, PD e PM6. Concluiu-se que práticas de manejo alimentar devem ser utilizadas para reduzir os efeitos dos fatores ambientais, como ano de nascimento, tipo de nascimento e peso da matriz ao parto, no desenvolvimento corporal dos cordeiros. Fatores de ajuste para sexo do cordeiro, tipo de nascimento e idade da ovelha devem ser estimados e considerados em programas de seleção, a fim de avaliar melhor o crescimento dos cordeiros da raça Morada Nova.

Palavras-chave: ovino deslanado, desenvolvimento corporal, fatores ambientais

\section{Environmental Effects on Growth Rate of Morada Nova Hair Lambs in Northeastern Brazil}

ABSTRACT - Records from an unselected flock of Morada Nova sheep collected over an 11-year period (1981 to 1991) were used to evaluate environmental sources of variation affecting growth traits. The effects of year of birth to yearling (A), sex of lamb (S), type of birth $(\mathrm{T})$, and weigth of ewe at lambing $(\mathrm{P})$ were important sources of variation to explain differences in weigth at birth (PN), weaning (PD), six- months (PM6) and 12 months (PM12) of age. Males were heavier than female lambs at all ages. Single lambs born and raised as singles (SS) weighed more at all ages than twins raised as singles (GS) or twins raised as twins (GG). Age of ewe at lambing (I) had a significant effect on PN, PD, and PM6. Results suggest that nutritional management should be improved to reduce the environmental effects such as year of birth, type of birth and weight of ewe lambing. Adjustment factors for sex of lamb, type of birth/rearing and age of ewe at lambing need to be estimated and considered in selection programs to improve growth performance of Morada Nova lambs.

Key Words: hair sheep, growth traits, environmental factors

\section{Introdução}

Os ovinos Morada Nova constituem uma das principais raças nativas de ovinos deslanados do Nordeste do Brasil. São explorados para carne e pele, sendo esta altamente apreciada no mercado internacional. Por serem animais de pequeno porte e bem adaptados às condições climáticas do semiárido, são importantes nas pequenas propriedades, onde constituem fonte de proteína na alimentação da população rural.

O peso corporal e a sobrevivência das crias são aspectos de significância econômica na criação (BRADFORD, 1985), sendo muito importante o conhecimento das diversas fases de cres- cimento que são consideradas na seleção animal. Programas de seleção e/ou de cruzamentos para melhoria da eficiência na produção requerem o conhecimento do crescimento dos animais, bem como a influência dos efeitos genéticos e ambientais sobre o mesmo.

Apesar da importância da ovinocultura no Nordeste, poucos estudos têm sido feitos em relação à caracterização e ao desenvolvimento corporal da raça Morada Nova. Os objetivos deste trabalho foram estudar o desenvolvimento corporal dos cordeiros da raça Morada Novapelagem vermelha, do nascimento até um ano de idade, e avaliar a importância dos fatores ambientais que influenciam o crescimento.

\footnotetext{
1 Professor do Instituto CENTEC e Professor Adjunto da Faculdade de Veterinária, Universidade Estadual do Ceará. E.mail: oria@centec.org.br 2 Professor Titular, Oklahoma State University - USA.

${ }^{3}$ Professor Adjunto, Depto de Zootecnia/UFC. E.mail: selaive@ufc.br
} 


\section{Material e Métodos}

Os dados foram provenientes dos registros de nascimento de cordeiros na Fazenda Experimental Iracema situada no município de Quixadá, Estado do Ceará e pertencente à Empresa de Pesquisa Agropecuária do Estado do Ceará-EPACE. Foram utilizadas 2755 informações de pesos ao nascer de cordeiros Morada Nova, no período de 1981 a 1990, provenientes de 806 diferentes ovelhas. As matrizes foram acasaladas com um total de 76 carneiros, com número desigual de reprodutores utilizados em cada ano. O nascimento dos cordeiros ocorreu nos meses de abril e maio e o desmame, de agosto a setembro, com aproximadamente 112 dias de idade. As práticas de manejo foram similares em todos os anos de observação, sendo os cordeiros mantidos com as ovelhas em pastagem nativa (caatinga) até o desmame e, posteriormente, separados por sexo em piquetes diferentes, porém em condições similares de manejo e alimentação até completarem um ano de idade.

Os dados para avaliar o desenvolvimento corporal foram: peso ao nascimento (PN), peso ao desmame (PD), peso aos seis meses de idade (PM6) e peso a um ano de idade (PM12). Ajustes individuais de PD, PM6 e PM12 para 112, 180 e 365 dias, respectivamente, foram efetuados antes das análises usando equações para cada peso em função das idades estudadas. Do total dos 2755 cordeiros nascidos, $1020(37 \%)$ nasceram de parto duplo e apenas 27 (menos de 1\%), de parto triplo, razão pela qual estes últimos foram considerados, para efeito de análises estatístico, como duplos.

O número de cordeiros estudados, nas diferentes faixas etárias avaliadas, é apresentado na Tabela 1. Os dados de peso corporal foram analisados pelo método dos quadrados mínimos para dados com freqüências desiguais nas subclasses, segundo os programas LSMLMW e MIXDML (HARVEY, 1990). O modelo linear utilizado para avaliar os fatores ambientais que influenciam os diferentes pesos corporais foi o seguinte:

$$
\begin{gathered}
Y_{i j k l m n}=\mu+R_{i}+A_{j}+S_{k}+T_{l}+I_{m}+A T_{j l}+S T_{k l} \\
+I T_{m l}+P_{n}+E_{i j k l m n}
\end{gathered}
$$

em que: $Y_{\text {ijklmn }}=$ valor observado para peso ao nascimento (PN), peso ao desmame (PD), peso aos 6 meses (PM6) e peso aos 12 meses (PM12) de idade do animal $n$, do sexo $k$, oriundo do tipo de parto $l$, filho do reprodutor $i$ e da ovelha com a idade $m$, no ano $j$;
Tabela 1 - Número de cordeiros estudados nas diferentes idades do desenvolvimento corporal, segundo o ano considerado

Table 1 - Number of lambs at different growth traits, according to year of birth

\begin{tabular}{lcccc}
\hline $\begin{array}{l}\text { Ano } \\
\text { Year }\end{array}$ & \multicolumn{4}{c}{$\begin{array}{c}\text { Número de cordeiros } \\
\text { Number of lambs }\end{array}$} \\
\cline { 2 - 5 } & $\begin{array}{c}\text { Nascimento } \\
\text { Birth }\end{array}$ & $\begin{array}{c}\text { Desmame } \\
\text { Weaning }\end{array}$ & $\begin{array}{c}\text { S meses } \\
\text { Six-month }\end{array}$ & $\begin{array}{c}1 \text { ano } \\
1 \text { year }\end{array}$ \\
\hline $1980 / 1981$ & 96 & 82 & 81 & 79 \\
$1981 / 1982$ & 260 & 214 & 196 & 184 \\
$1982 / 1983$ & 305 & 262 & 240 & 222 \\
$1983 / 1984$ & 357 & 283 & 243 & 223 \\
$1984 / 1985$ & 320 & 237 & 209 & 178 \\
$1985 / 1986$ & 359 & 291 & 251 & 192 \\
$1986 / 1987$ & 206 & 172 & 150 & 87 \\
$1987 / 1988$ & 367 & 290 & 261 & 87 \\
$1988 / 1989$ & 274 & 248 & 223 & 120 \\
$1989 / 1990$ & 211 & 188 & 114 & 79 \\
Total & 2755 & 2267 & 1968 & 1451 \\
\hline
\end{tabular}

$\mu=$ média geral da característica em estudo; $R_{i}=$ efeito aleatório de reprodutor $i ; A_{j}=$ efeito fixo do ano de nascimento $j(j=1, \ldots \ldots, 10) ; S_{k}=$ efeito fixo do sexo da cria $k(1=$ macho, $2=$ fêmea $) ; T_{l}=$ efeito fixo do tipo de nascimento/criação $l$ do cordeiro (para PN: $\mathrm{S}=$ simples, D = duplo; para PD, PM6 e PM12: $\mathrm{SS}=$ cordeiro nascido simples e criado simples; GS = cordeiro nascido duplo e criado simples; $\mathrm{GG}=$ cordeiro nascido duplo e criado como duplo); $I_{m}=$ efeito fixo da classe da idade da mãe $m$ (classe $1=$ ovelhas $<2$ anos; classe $2=2$ a $<3$ anos; classe $3=3$ a $<4$ anos, ......; classe $6=>6$ anos; $(A T)_{i l}$; $(S T)_{k l} ;(I T)_{m l}=$ interações duplas entre os efeitos anteriores; $P_{n}=$ efeito do peso da ovelha ao parto (covariável); $E_{i j k l m n o}=$ erro aleatório, normal, independentemente distribuído, com média zero e variância $\sigma^{2}$.

\section{Resultados e Discussão}

De acordo com o resultado da análise de variância apresentado na Tabela 2, o peso corporal ao nascimento (PN), ao desmame (PD), aos seis meses (PM6) e aos 12 meses de idade (PM12) foi influenciados $(\mathrm{P}<0,01)$ pelos efeitos de reprodutor, ano do nascimento (A), sexo do cordeiro (S), tipo de nascimento (T), idade da ovelha (I) e peso da ovelha ao parto (PO). Das interações estudadas, AxT (ano de nascimento $\mathrm{x}$ tipo de nascimento) foi significativa $(\mathrm{P}<0,01)$ para PD, PM6 e PM12, enquanto IxT (idade 
da ovelha $\mathrm{x}$ tipo de nascimento) foi significativa $(\mathrm{P}<0,05)$ somente para PD e PM6.

As médias de peso corporal ajustadas pelo método dos quadrados mínimos, segundo o ano de nascimento, sexo do cordeiro, tipo de nascimento e a idade da ovelha, são apresentadas na Tabela 3. A média geral do PN, baseada em 2755 observações, foi de 2,21 \pm $0,01 \mathrm{~kg}$, com variações significativas entre anos. Cordeiros machos foram mais pesados $(\mathrm{P}<0,01)$ ao nascimento do que as fêmeas $(2,26$ vs $2,16 \mathrm{~kg})$, da mesma forma que os cordeiros nascidos de parto simples foram significativamente $(\mathrm{P}<0,01)$ mais pesados $(2,44 \pm 0,02 \mathrm{~kg})$ que os nascidos de partos duplos $(1,97 \pm 0,02 \mathrm{~kg})$.

$\mathrm{O}$ peso ao nascimento aumentou com a idade das ovelhas, observando-se os cordeiros mais leves nas matrizes com idade inferior a 2 anos (Tabela 3). Por outro lado, o peso corporal das ovelhas ao parto (P) influenciou significativamente $(\mathrm{P}<0,05)$ o $\mathrm{PN}$ dos cordeiros, sendo o coeficiente de regressão linear do $\mathrm{P}$ para $\mathrm{PN}$ de $0,03 \pm 0,0$.

O peso médio dos cordeiros ao desmame (PD), baseado em 2267 observações, foi de 10,93 \pm 0,09 kg, com diferenças significativas $(\mathrm{P}<0,01)$ entre anos (Tabela 2). Os cordeiros machos foram $0,31 \mathrm{~kg}$ mais pesados ao desmame que as cordeiras fêmeas. $\mathrm{O}$ contraste linear entre as médias de peso ao desmame dos cordeiros nascidos de parto simples e criados como únicos (SS), em relação aos de partos duplos nascidos e criados como gêmeos (GG) e/ou criados como único (GS), foi significativo $(\mathrm{P}<0,05)$. Outro contraste mostrou que cordeiros GS tiveram maior $\mathrm{PD}(\mathrm{P}<0,05)$ do que os $\mathrm{GG}$.

A idade da ovelha influenciou significativamente $(\mathrm{P}<0,05)$ o $\mathrm{PD}$, variando de $10,58 \pm 0,20 \mathrm{~kg}$ para cordeiros nascidos de ovelhas acima de 6 anos a $11,30 \pm 0,13 \mathrm{~kg}$ para os nascidos de ovelhas de 2 a 3 anos de idade. O peso da ovelha ao parto influenciou também significativamente $(\mathrm{P}<0,05)$ o $\mathrm{PD}$. A regressão linear do $\mathrm{PD}$ em relação ao $\mathrm{P}$ foi significativa positiva e igual a $0,14 \pm 0,01$.

Observou-se também interação significativa entre AxT $(\mathrm{P}<0,01)$. Os cordeiros GG nascidos em 1983, 1984 e 1985 apresentaram os menores pesos ao desmame $(8,22 \pm 0,27,8,31 \pm 0,27$ e $8,40 \pm 0,35$, respectivamente), enquanto os nascidos como SS em 1981 e 1990 foram os mais pesados $(13,39 \pm 0,48$ e $13,23 \pm 0,27 \mathrm{~kg}$, respectivamente).

A interação IxT também influenciou significativamente o PD. A análise de contraste linear mostrou que cordeiros SS nascidos de ovelhas de $<2$ e $>6$ anos foram significativamente $(\mathrm{P}<0,05)$ mais leves que os nascidos de ovelhas com idade entre $>2$ e $<6$ anos. Entretanto, cordeiros GG nascidos de ovelhas dos diferentes grupos

Tabela 2 - Análise de variância para os pesos corporais dos cordeiros nas diferentes idades de crescimento Table 2 - Analysis of variance for body weigth of lambs at different growing ages

\begin{tabular}{|c|c|c|c|c|c|c|c|c|}
\hline \multirow[t]{2}{*}{$\begin{array}{l}\text { Fontes de variação } \\
\text { Sources of variation }\end{array}$} & \multicolumn{2}{|c|}{$\begin{array}{c}\text { Peso ao } \\
\text { nascer } \\
\text { Birth } \\
\text { weigth }\end{array}$} & \multicolumn{2}{|c|}{$\begin{array}{c}\text { Peso ao } \\
\text { desmame } \\
\text { Weaning } \\
\text { weigth }\end{array}$} & \multicolumn{2}{|c|}{$\begin{array}{c}\text { Peso aos } \\
6 \text { meses } \\
6 \text { - month } \\
\text { weigth }\end{array}$} & \multicolumn{2}{|c|}{$\begin{array}{c}\text { Peso aos } \\
12 \text { meses } \\
12-\text { month } \\
\text { weigth }\end{array}$} \\
\hline & GL & $\mathrm{QM}$ & GL & $\mathrm{QM}$ & GL & $\mathrm{QM}$ & GL & QM \\
\hline $\begin{array}{l}\text { Reprodutor } \\
\text { Sire of lamb }\end{array}$ & 75 & $0,2773 * *$ & 75 & $4,3233 * *$ & 75 & $4,9743 *$ & 75 & $9,4939 * *$ \\
\hline $\begin{array}{l}\text { Ano nascimento (A) } \\
\text { Year of birth }\end{array}$ & 9 & $0,6942 * *$ & 9 & $62,9884 * *$ & 9 & $19,1608 * *$ & 9 & $36,7845^{* *}$ \\
\hline $\begin{array}{l}\text { Sexo do cordeiro (S) } \\
\text { Sex of lamb }\end{array}$ & 1 & $4,9600 * *$ & 1 & $26,0340 * *$ & 1 & $34,5016^{* *}$ & 1 & $72,1670 * *$ \\
\hline $\begin{array}{l}\text { Tipo nascimento/criação }(\mathrm{T}) \\
\text { Type birth/rearing }\end{array}$ & ) & $82,2856^{* *}$ & 2 & $743,2329 * *$ & 2 & $539,2475 * *$ & 2 & $233,6548 * *$ \\
\hline $\begin{array}{l}\text { Idade da ovelha (I) } \\
\text { Age of ewe }\end{array}$ & 5 & $1,0972 * *$ & 5 & $7,3685^{*}$ & 5 & $8,28497 *$ & 5 & 10,6413 \\
\hline $\mathrm{A} \times \mathrm{T}$ & 9 & 0,1756 & 18 & $17,7416^{* *}$ & 18 & $21,3221 * *$ & 18 & $14,9540 * *$ \\
\hline $\mathrm{S} \times \mathrm{T}$ & 1 & 0,2253 & 2 & $6,7183+$ & 2 & 0,3459 & 2 & 1,9184 \\
\hline $\mathrm{I} \times \mathrm{T}$ & 5 & $0,3930+$ & 10 & $6,5055^{*}$ & 10 & $7,4931 *$ & 10 & $10,4077+$ \\
\hline $\begin{array}{l}\text { Peso da ovelha ao parto } \\
\text { Weigth of ewe at lambing }\end{array}$ & 1 & $18,9678 * *$ & 1 & $380,8335^{*}$ & 1 & $476,3198 * *$ & 1 & $388,4506^{* *}$ \\
\hline $\begin{array}{l}\text { Erro experimental } \\
\text { Error }\end{array}$ & 2647 & 0,1866 & 2146 & 2,8464 & 1844 & 3,6267 & 1327 & 5,8814 \\
\hline
\end{tabular}

$+\mathrm{P}<0,10 ;{ }^{*} \mathrm{P}<0,05 ;{ }^{* \star} \mathrm{P}<0,01$ 
de idade apresentaram PD similares.

A média de peso dos cordeiros aos seis meses de idade (PM6) foi de 12,80 $\pm 0,11 \mathrm{~kg}$, com variações entre anos de $11,45 \pm 0,28 \mathrm{~kg}(1984)$ a $13,98 \pm 0,36 \mathrm{~kg}$ (1987), conforme observa-se na Tabela 2. Nesta idade, os cordeiros machos foram mais pesados que as fêmeas $(13,00 \pm 0,13 \mathrm{~kg}$ vs $12,60 \pm 0,13 \mathrm{~kg})$. Os cordeiros SS atingiram, em média, $14,08 \pm 0,10 \mathrm{~kg}$, enquanto os cordeiros GS e GG pesaram, respectivamente, $12,65 \pm 0,24$ e $11,68 \pm 0,14 \mathrm{~kg}$.

Os PM6 dos cordeiros aumentaram de $12,83 \pm 0,18 \mathrm{~kg}$ para os nascidos de ovelhas $<2$ anos à $13,24 \pm 0,15 \mathrm{~kg}$ para os oriundos de matrizes de $>3$ anos de idade. $\mathrm{O}$ peso da ovelha ao parto também teve efeito significativo sobre PM6 ( $b=0,17 \pm 0,01)$, com influência significativa da interação IxT. Cordeiros SS nascidos de ovelhas de $<2$ e $>6$ anos $(13,70 \pm 0,13$ e 13,41 $\pm 0,25 \mathrm{~kg}$, respectivamente) apresentaram menor PM6 que os cordeiros nascidos de ovelhas entre $>2$ e $<6$ anos $(14,11 \pm 0,21$ a $14,46 \pm 0,14 \mathrm{~kg})$. Por outro lado, o PM6 dos cordeiros GG nascidos de ovelhas com idade superior a cinco anos foram mais leves que os nascidos de ovelhas com menor idade.

Os cordeiros ao atingirem um ano de idade (PM12) tiveram média de peso corporal de $18,60 \pm 0,20 \mathrm{~kg}$. As fontes significativas de variação para PM12 foram

Tabela 3 - Médias e erros-padrões para pesos corporais dos cordeiros nas diferentes idades de crescimento. Período 1981-1990

Table 3 - Means and standard errors for live body weigth of lambs at different growing ages. Period 1981-1990

\begin{tabular}{|c|c|c|c|c|c|c|c|c|}
\hline \multirow[t]{2}{*}{$\begin{array}{l}\text { Efeitos } \\
\text { Factors }\end{array}$} & \multicolumn{2}{|c|}{$\begin{array}{c}\text { Peso ao } \\
\text { nascer }(\mathrm{kg}) \\
\text { Birth } \\
\text { weigth }\end{array}$} & \multicolumn{2}{|c|}{$\begin{array}{c}\text { Peso ao } \\
\text { desmame }(\mathrm{kg}) \\
\text { Weaning } \\
\text { weigth }\end{array}$} & \multicolumn{2}{|c|}{$\begin{array}{c}\text { Peso aos } \\
6 \text { meses }(\mathrm{kg}) \\
6 \text { - month } \\
\text { weigth }\end{array}$} & \multicolumn{2}{|c|}{$\begin{array}{c}\text { Peso ao } \\
1 \text { ano }(\mathrm{kg}) \\
1 \text { year } \\
\text { weigth }\end{array}$} \\
\hline & $\mathrm{N}^{\mathrm{O}}$ & Média \pm EP & $\mathrm{N}^{\mathrm{O}}$ & Média \pm EP & $\mathrm{N}^{\mathrm{O}}$ & $\overline{\text { Média } \pm \mathrm{EP}}$ & $\mathrm{N}^{\mathrm{O}}$ & Média \pm EP \\
\hline $\begin{array}{l}\text { Média geral } \\
\text { Overall mean }\end{array}$ & 2755 & $2,21 \pm 0,01$ & 2267 & $10,93 \pm 0,09$ & 1968 & $12,80 \pm 0,11$ & 1451 & $18,60 \pm 0,20$ \\
\hline $\begin{array}{l}\text { Ano nascime } \\
\text { Year of birth }\end{array}$ & & & & & & & & \\
\hline 1981 & 96 & $2,30 \pm 0,11$ & 82 & $11,72 \pm 0,56$ & 81 & $13,67 \pm 0,65$ & 79 & $20,41 \pm 0,89$ \\
\hline 1982 & 260 & $2,40 \pm 0,09$ & 214 & $11,88 \pm 0,41$ & 196 & $12,80 \pm 0,48$ & 184 & $17,66 \pm 0,67$ \\
\hline 1983 & 305 & $2,34 \pm 0,05$ & 262 & $10,00 \pm 0,25$ & 240 & $12,19 \pm 0,31$ & 222 & $16,49 \pm 0,44$ \\
\hline 1984 & 357 & $2,34 \pm 0,05$ & 283 & $9,23 \pm 0,22$ & 243 & $11,45 \pm 0,28$ & 223 & $17,66 \pm 0,41$ \\
\hline 1985 & 320 & $2,06 \pm 0,05$ & 237 & $9,27 \pm 0,23$ & 209 & $12,06 \pm 0,30$ & 178 & $18,87 \pm 0,42$ \\
\hline 1986 & 359 & $2,07 \pm 0,04$ & 291 & $10,17 \pm 0,21$ & 251 & $12,59 \pm 0,27$ & 192 & $17,97 \pm 0,43$ \\
\hline 1987 & 206 & $2,04 \pm 0,05$ & 172 & $12,82 \pm 0,29$ & 150 & $13,98 \pm 0,36$ & 87 & $20,23 \pm 0,63$ \\
\hline 1988 & 367 & $2,14 \pm 0,04$ & 290 & $11,66 \pm 0,22$ & 261 & $13,22 \pm 0,26$ & 87 & $19,42 \pm 0,51$ \\
\hline 1989 & 274 & $2,15 \pm 0,05$ & 248 & $10,84 \pm 0,28$ & 223 & $12,99 \pm 0,35$ & 120 & $19,73 \pm 0,64$ \\
\hline 1990 & 211 & $2,23 \pm 0,06$ & 188 & $11,71 \pm 0,39$ & 144 & $13,07 \pm 0,47$ & 79 & $17,58 \pm 0,84$ \\
\hline
\end{tabular}

Sexo do cordeiro

Sex of lamb

Macho

1416

$2,26 \pm 0,02$

1151

$11,09 \pm 0,11 \quad 968$

$13,00 \pm 0,13$

676

$18,95 \pm 0,23$

1339

$2,16 \pm 0,02 \quad 1116$

$10,78 \pm 0,11$

1339

$12,60 \pm 0,13$

775

$18,25 \pm 0,23$

Female

Tipo de nascimento/criação

Type of birth/rearing

Simples criado único

Gêmeo criado gêmeo

Gêmeo criado simples

1708 1047

$2,44 \pm 0,02$

$1,97 \pm 0,02$

1530

546

191

$12,33 \pm 0,08$
$9,83 \pm 0,12$
$10,64 \pm 0,19$

1378

441

149

$14,08 \pm 0,10$

$11,68 \pm 0,14$

$12,65 \pm 0,24$

1.074

Idade da ovelha

Age of ewe

1 até $<2$ anos

2 até $<3$ anos

3 até $<4$ anos

4 até $<5$ anos

5 até $<6$ anos

$>6$ anos
661
660
576
414
255
189

545

$2,21 \pm 0,03$

$2,20 \pm 0,03$

$2,22 \pm 0,03$
$2,25 \pm 0,02$

$2,25 \pm 0,02$
$10,92 \pm 0,15 \quad 483$

$11,30 \pm 0,13 \quad 515$

$10,97 \pm 0,13 \quad 413$

$10,96 \pm 0,15 \quad 283$

$10,85 \pm 0,18 \quad 160$

$10,58 \pm 0,20 \quad 114$
$12,83 \pm 0,18$

$13,24 \pm 0,15$

$12,86 \pm 0,16$

$12,86 \pm 0,19$

$12,64 \pm 0,24$

$12,38 \pm 0,26$

\begin{tabular}{cc}
383 & $18,67 \pm 0,30$ \\
387 & $19,00 \pm 0,25$ \\
294 & $18,45 \pm 0,27$ \\
205 & $19,09 \pm 0,32$ \\
106 & $18,25 \pm 0,44$ \\
76 & $18,15 \pm 0,41$ \\
\hline
\end{tabular}


ano de nascimento, sexo da cria, tipo de nascimento, peso da ovelha ao parto e a interação AxT. A idade da ovelha (I) e as interações SxT e IxT não influenciaram significativamente $(\mathrm{P}>0,05) \mathrm{PM} 12$ (Tabela 2).

O PM12 dos cordeiros machos foi mais pesado $(\mathrm{P}<0,01)$ do que as fêmeas, com peso superior em $0,70 \mathrm{~kg}$, em média $(18,95 \pm 0,23$ e $18,25 \pm 0,23 \mathrm{~kg}$, respectivamente) e os cordeiros $\mathrm{SS}$ foram mais pesados $(19,66 \pm 0,18 \mathrm{~kg}$ ( que os cordeiros GG e GS $(17,63 \pm 0,26$ e $18,51 \pm 0,41 \mathrm{~kg}$, respectivamente). Os contrastes lineares do PM12 dos cordeiros SS em relação aos GG e GS e entre cordeiros GS e GG foram significativos. A regressão linear do PM12 em relação ao peso da ovelha ao parto (PO) foi também significativamente positiva e igual a $0,17 \pm 0,02$.

Os resultados descritos mostram que os fatores ambientais afetam o crescimento dos cordeiros da raça Morada Nova, do nascimento a um ano de idade. O efeito ano foi um fator importante em todas as fases do crescimento dos cordeiros. A influência do ano é um fator esperado pelo relatado na literatura (DICKERSON e LASTER, 1975; KAUSHISH et al., 1990; BUVANENDRAN et al., 1992), bem como nas condições climáticas do Nordeste do Brasil. A influência do ano se produz por diversas ocorrências como a quantidade e distribuição das chuvas, problemas sanitários, práticas de manejo e disponibilidade de forragens. Durante o período experimental, a pluviosidade apresentou grandes variações entre os anos e dentre os meses do ano, incluindo três anos de seca, que certamente contribuíram para ressaltar ainda mais o efeito ano. Outro aspecto a considerar é a disponibilidade e deterioração da forragem através dos anos, considerando que a área experimental foi a mesma durante todo o período.

O peso corporal dos cordeiros observado nas diferentes fases do crescimento foi considerado muito baixo, quando comparado com o desenvolvimento de ovinos de clima temperado. Problemas de temperatura e alimentação podem ser considerados como fatores essenciais. Na região semi-árida do Brasil, os animais são mantidos a maior parte do ano somente em pastagens nativa, insuficiente para suprir as necessidades nutricionais, principalmente durante a época seca. Contudo, o maior peso observado dos machos sobre as fêmeas, em todas as fases de crescimento estudadas, concordam com os resultados obtidos por outros autores (FERNANDES, 1985; PEREIRA et al., 1987; OLIVEIRA, 1992). A superioridade no peso corporal dos machos é devida ao dimorfismo sexual comum a todas as espécies mamíferas na fase de desenvolvimento e na idade adulta. Porém, apesar da diferença significativa observada $(\mathrm{P}<0,01)$, o maior peso dos machos pode ser considerado pequeno em relação ao dos outros trabalhos consultados. Uma possível explicação para a pouca diferença encontrada é que, além das condições precárias de nutrição, o desmame dos cordeiros coincidiu com o inicio da época seca, conseqüentemente, os cordeiros machos não tiveram oportunidade para expressar seu maior potencial de crescimento.

Os resultados sobre o efeito do tipo de nascimento e/ou parto (T) sobre os pesos corporais, observados em todas as fases de crescimento neste trabalho, estão de acordo com os trabalhos publicados na literatura (MARTINEZ, 1983, MOLINA et al., 1991). Em geral, é esperado que cordeiros nascidos como simples (SS) apresentem maior crescimento que os nascidos como duplo e criados como simples (GS) e estes, por sua vez, cresçam mais que os nascidos e criados como duplos (GG). Este fato é devido à competição por leite. A maior disponibilidade de leite materno para os cordeiros SS e GS retarda o início do consumo de pasto em relação aos cordeiros GG, fato que pode explicar em parte as desvantagens dos cordeiros GG. Todavia, provavelmente pelo fato de terem nascidos mais leves, os cordeiros múltiplos apresentaram ganho de peso compensatório após o desmame, que fez diminuir proporcionalmente as diferenças observadas ao desmame, porém sem igualar-se aos nascidos de parto simples.

\section{Conclusões}

Fatores de ajuste como sexo do cordeiro, tipo de nascimento e idade da ovelha na parição devem ser estimados e considerados em programas de seleção, a fim de avaliar melhor o crescimento dos cordeiros da raça Morada Nova.

Práticas de manejo alimentar devem ser utilizadas para reduzir os efeitos dos fatores ambientais, como ano de nascimento, tipo de parto e peso da matriz ao parto, no desenvolvimento corporal dos cordeiros.

\section{Referências Bibliográficas}

BRADFORD, G.F. 1985. Selection for litter size. In: LAND, R.B., ROBINSON, D.W.(Eds.). Genetics of reproduction in sheep. Butterworths, London. p.3-18.

BUVANENDRAN, V., MAKUZA, S.M., CHIRONGA, P. 1992. Phenotypic and genetic parameters of weaning traits in Dorper sheep in Zimbabwe. Small Rum. Res., 7(4):369-374.

DICKERSON, G.E., LASTER, D.B. 1975. Breed, heterosis and 
environmental influences on growth and puberty in ewe lambs. J. Anim. Sci., 41:1-9.

FALCONER, D.S. 1989. Introduction to quantitative genetics. Longman, Inc. 3.ed. New York.

FERNANDES, A.A.O. Genetic and environmental factors affecting growwth and reproduction characters of Morada Nova sheep in Northeast Brazil. Texas: Texas A \& M Univ., College Station, 1985 ( M.S.Thesis).

HARVEY, W.R. User's Guide for LSMLMW and MIXMDL., Mixed model least-squares and maximum likelihood computer program. Columbus: Ohio State University, USA, 1990 (Mimeo).

KAUSHISH, S.K., RAWAT, P.S., SHARMA, C. 1990. Performance of native sheep (Malpua) and its crosses with Avikaline under semi-arid conditions. World Rev. Anim. Prod., 1:43-45.

MARTINEZ, A. 1983. Reproduction and growth of hair sheep in an experimental flock in Venezuela. In: FITZHUGH, H.A., BRADFORD, G.E. (Eds). Hair sheep of Western Africa and the Americas: a genetic resouce for the tropics. Westview Press, Boulder, Colorado. p.105-117.
MOLINA, A., GALlEGO, L., PEREZ, J.I., BERNABEU, R. 1991. Crescimiento de corderos de raza Manchega según la condición corporal da las ovejas, la época del parto, el tipo de parto y el sexo. Avances en Alimentación y Mejora Animal, 31:198.

OLIVEIRA, S.M.P. Desempenho de ovinos da Raça Morada Nova variedade branca no Estado do Ceará: Parâmetros genéticos e de ambiente. Belo Horizonte, BH: UFMG. Dissertação (Mestrado em Zootecnia) - Universidade Federal de Minas Gerais, 1992. 69p.

PEREIRA, R.M.A., LIMA, F.A.M., FREITAS, J.P., SILVA, M.A. 1987. Heredabilidade de peso e desempenho do nascimento aos 360 dias de idade de ovinos da raça Morada Nova variedade branca no Estado do Ceará. R. Soc. Bras. Zootec., 16(5):402-410.

Recebido em: 29/04/98

Aceito em: 31/05/01 